\title{
Comparing Syndromic Data to Discharge Data to Measure Opioid Overdose Emergency Department Visits
}

\author{
Emilia S. Pasalic, Alana M. Vivolo-Kantor, Pedro Martinez
}

CDC National Center for Injury Prevention and Control (NCIPC), Atlanta, Georgia, United States

Objective

Epidemiologists will understand the differences between syndromic and discharge emergency department data sources, the strengths and limitations of each data source, and how each of these different emergency department data sources can be best applied to inform a public health response to the opioid overdose epidemic.

\section{Introduction}

Timely and accurate measurement of overdose morbidity using emergency department (ED) data is necessary to inform an effective public health response given the dynamic nature of opioid overdose epidemic in the United States. However, from jurisdiction to jurisdiction, differing sources and types of ED data vary in their quality and comprehensiveness. Many jurisdictions collect timely emergency department data through syndromic surveillance (SyS) systems, while others may have access to more complete, but slower emergency department discharge datasets. State and local epidemiologists must make decisions regarding which datasets to use and how to best operationalize, interpret, and present overdose morbidity using ED data. These choices may affect the number, timeliness, and accuracy of the cases identified.

\section{Methods}

CDC partnered with 45 states and the District of Columbia to combat the worsening opioid overdose epidemic through three cooperative agreements: Prevention for States (PFS), Data Driven Prevention Initiative (DDPI), and Enhanced State Opioid Overdose Surveillance (ESOOS). To support funded jurisdictions in monitoring non-fatal opioid overdoses, CDC developed two different sets of indicator guidance for measuring non-fatal opioid overdoses using ED data, with each focusing on different ED data sources (SyS and discharge). We report on the following attributes for each type of ED data source [1,2]: 1) timeliness; 2) data quality (e.g., percent completeness by field); 3) validity; and 4) representativeness (e.g., percent of facilities included).

\section{Results}

When comparing timeliness across data sources, SyS data has clear advantages, with many jurisdictions receiving data within 24 hours of an event. For discharge data, timeliness is more variable with some jurisdictions receiving data within weeks while others wait over 1.5 years before receiving a complete discharge dataset. Data quality and completeness tends to be stronger in discharge datasets as facilities are required to submit complete discharge records with valid ICD-10-CM codes in order to be reimbursed by payers. By contrast, for SyS data systems, participating facilities may not consistently submit data for all possible fields, including diagnosis. Validity is dependent on the data source as well as the case definition or syndrome definition used; with this in mind, SyS data overdose indicators are designed to have high sensitivity, with less attention to specificity. Discharge data overdose indicators are designed to have a high positive predictive value, while sensitivity and specificity are both important considerations. Discharge datasets often include records for $100 \%$ of ED visits from all nonfederal, acute care-affiliated facilities in a state included. By contrast, representativeness of facilities in SyS data systems varies widely across states with some states having less than $50 \%$ of facilities reporting.

\section{Conclusions}

CDC funded partners share overdose morbidity data with CDC using either ED SyS data, ED discharge data, or both. CDC indicator guidance for ED discharge data is designed for states to track changes in health outcomes over time for descriptive, performance monitoring, and evaluation purposes and to create rates that are more comparable across injury category, time, and place. Considering these objectives, CDC placed a higher priority on data quality, validity (i.e., positive predictive value), and representativeness, all of which are stronger attributes of discharge data. CDC's indicator guidance for ED SyS data is designed for states to rapidly identify changes in nonfatal overdoses and to identify areas within a particular state that are experiencing rapid change in the frequency or types of overdose events. When considering these needs, CDC prioritized timeliness and validity in terms of sensitivity, both of which are stronger attributes of SyS data. SyS and discharge ED data each lend themselves to different informational applications and interpretations based on the strengths and limitations of each dataset. An effective, informed public

ISDS Annual Conference Proceedings 2019. This is an Open Access article distributed under the terms of the Creative Commons AttributionNoncommercial 4.0 Unported License (http://creativecommons.org/licenses/by-nc/3.0/), permitting all non-commercial use, distribution, and reproduction in any medium, provided the original work is properly cited. 
ISDS 2019 Conference Abstracts

health response to the opioid overdose epidemic requires continued investment in public health surveillance infrastructure, careful consideration of the needs of the data user, and transparency regarding the unique strengths and limitations of each dataset.

\section{Acknowledgement}

The authors would like to acknowledge CDC's state and jurisdictional partners funded through the ESOOS, PFS, and DDPI cooperative agreements; CDC staff from CSELS, NCHS, and NCIPC; and the CSTE ICD-10-CM Drug Poisoning Indicators Workgroup for their engagement, comments, and brilliant epidemiological insight during the indicator development and testing processes. The findings and conclusions in this report are those of the authors and do not necessarily represent the official position of the Centers for Disease Control and Prevention.

\section{References}

1. Pencheon D. (2006). Oxford handbook of public health practice. 2nd ed. Oxford: Oxford University Press.

2. Centers for Disease Control and Prevention (CDC) Evaluation Working Group on Public Health Surveillance Systems for Early Detection of Outbreaks. (May 7, 2004). Framework for Evaluating Public Health Surveillance Systems for Early Detection of Outbreaks. MMWR. Morbidity and Mortality Weekly Reports. Retrieved from:

https://www.cdc.gov/mmwr/preview/mmwrhtml/rr5305a1.htm

Figure 1. Comparing Syndromic Data to Discharge Data to Measure Opioid Overdose Emergency Department Visits

Timeliness
Data Quality
Validity
Representativeness

\section{ED SyS data}

Faster

Lower

Varies

Varies

\author{
ED Discharge Data \\ Varies \\ Higher \\ Varies \\ Higher
}

